6-1997

\title{
Symmetry Breaking in Crossed Magnetic and Electric Fields
}

C. Neumann

R. Ubert

S. Freund

et al.

John B. Delos

William \&Mary, jbdelos@wm.edu

Follow this and additional works at: https://scholarworks.wm.edu/aspubs

Part of the Physics Commons

\section{Recommended Citation}

Neumann, C.; Ubert, R.; Freund, S.; al., et; and Delos, John B., Symmetry Breaking in Crossed Magnetic and Electric Fields (1997). Physical Review Letters, 78(25), 4705-4708.

https://doi.org/10.1103/PhysRevLett.78.4705

This Article is brought to you for free and open access by the Arts and Sciences at W\&M ScholarWorks. It has been accepted for inclusion in Arts \& Sciences Articles by an authorized administrator of W\&M ScholarWorks. For more information, please contact scholarworks@wm.edu. 


\title{
Symmetry Breaking in Crossed Magnetic and Electric Fields
}

\author{
C. Neumann, R. Ubert, S. Freund, E. Flöthmann, B. Sheehy,* and K. H. Welge ${ }^{\dagger}$ \\ Fakultät für Physik, Universität Bielefeld, Universitätsstrasse 25, D-33615 Bielefeld, Germany \\ M. R. Haggerty and J. B. Delos ${ }^{\dagger}$ \\ Physics Department, College of William and Mary, Williamsburg, Virginia 23187
}

(Received 14 January 1997)

\begin{abstract}
We present the first observations of cylindrical symmetry breaking in highly excited diamagnetic hydrogen with a small crossed electric field, and we give a semiclassical interpretation of this effect. As the small perpendicular electric field is added, the recurrence strengths of closed orbits decrease smoothly to a minimum, and revive again. This phenomenon, caused by interference among the electron waves that return to the nucleus, can be computed from the azimuthal dependence of the classical closed orbits. [S0031-9007(97)03431-5]

PACS numbers: $32.60 .+\mathrm{i}, 03.65 . \mathrm{Sq}, 05.45 .+\mathrm{b}$
\end{abstract}

In 1969, Garton and Tomkins reported what they called "quasi-Landau resonances"-oscillatory modulations in the near-ionization absorption spectrum of barium in a strong magnetic field [1]. Edmonds analyzed these oscillations semiclassically by considering the motion of the Rydberg electron in the plane perpendicular to the magnetic field [2]. In this plane there is a simple family of closed orbits: classical electron orbits that start at the ionic core and, moving under the combined effect of the Coulomb and magnetic fields, eventually return to the core. He found that Bohr-Sommerfeld quantization of these orbits predicts energy level spacings equal to the period of the modulations seen in the experiment. Later experiments have demonstrated that the absorption spectra of highly excited atoms in various field configurations are influenced by many two- and three-dimensional closed orbits [3-6].

According to closed orbit theory [7,8], each classical orbit that starts at the atom carries along with it a portion of the outgoing electron wave. If such an orbit returns to the nucleus, its companion wave interferes with the steady stream of outgoing waves, and this interference produces an oscillation in the absorption spectrum. The return of the electron, and the resulting interference oscillation in the spectrum, is called a recurrence. The period of the oscillation is inversely proportional to the classical action of the closed orbit, and its strength is proportional to the electron current density in the neighborhood of that orbit. Thus, the more that orbits are focused on the atom, the stronger the associated oscillation in the spectrum, i.e., the stronger the recurrence.

Most of the experiments and theory to date have dealt with Rydberg atoms in static external fields along a single axis - magnetic [3], electric [4], or parallel electric and magnetic fields [5]. In such cylindrically symmetric systems, the angular momentum component $L_{z}$ (i.e., $p_{\phi}$ ) along the field axis $(\hat{\mathbf{z}})$ is conserved. Classical orbits are therefore organized into cylindrical families, with orbits within a family differing from one another only by a rotation about the $z$ axis. When orbits in a family return to the core, they do so in a perfect circular focus, giving a strong recurrence.

The dynamics is profoundly changed when the cylindrical symmetry is broken by crossing the magnetic field with an electric field. Now instead of a continuous family of classical orbits returning in perfect focus, only two isolated orbits return exactly to the origin. Meanwhile, the nonconservation of $p_{\phi}$ makes it impossible to separate the $\phi$ motion [9]. There have been several studies of recurrences in crossed fields [6], but none of them address the present issue: the transition from symmetry to asymmetry, i.e., from two to three nonseparable degrees of freedom. How the dynamics behaves under such symmetry changes and how it is described in semiclassical theory is a basic problem [10]. What is the signature of symmetry breaking in an atomic absorption spectrum?

In this Letter we report the first experiments on the dynamical evolution of Rydberg hydrogen atoms in a strong magnetic field when the cylindrical symmetry is broken by adding a small crossed electric field. We present results obtained for the Garton-Tomkins-Edmonds (GTE) recurrence, which shows the characteristic signature of symmetry breaking. (We have observed the same signature for other orbits [11].) We also present a quantitative semiclassical theory capable of describing this symmetry breaking, and use it to calculate the evolution of the recurrence strength.

Experiment.-The experiments are performed, as previously [12], with deuterium atoms in a beam collimated along the magnetic field axis, excited at the center of the crossed fields by pulsed lasers perpendicular to the atomic beam. The excitation occurs in two steps, $D(1 s)+$ $h \nu_{1} \rightarrow D(2 p)+h \nu_{2} \rightarrow D^{*}\left(n, m_{\ell}=0\right)$, to energies near the field-free ionization limit $(n \approx 62-112)$. $D^{*}$ atoms in long-lived states, proceeding at thermal velocity, are detected via electrons created by field ionization. Electrons from atoms promptly ionized within the interaction 
region are prevented from crossed-field drifting by turning off the crossed electric field quickly after the excitation, and are trapped there temporarily then released to the detector. The combined signal of the two electron groups is thus proportional to the total absorption cross section [11].

The Hamiltonian for hydrogen in crossed fields (in atomic units, $\hbar=e=m_{e}=1$ ) is

$H=\frac{1}{2} p^{2}-1 / r+\frac{1}{2} B L_{z}+\frac{1}{8} B^{2}\left(x^{2}+y^{2}\right)+F x$.

Because of a classical scaling law for this system, the shapes of classical orbits do not depend on the energy $E$, electric field strength $F$, and magnetic field strength $B$ separately, but only on the scaled energy $\epsilon \equiv E B^{-2 / 3}$ and scaled electric field $f \equiv F B^{-4 / 3}$. The classical action $S \equiv$ $\int \mathbf{p} \cdot d \mathbf{q}$ scales as $S=\tilde{S} w$, where $\tilde{S}$ is a scaled action that also depends only on $\epsilon$ and $f$, and $w \equiv B^{-1 / 3}$. We use the method of scaled variable recurrence spectroscopy [12]: we measure the photoabsorption cross section $\sigma$ as a function of $w$ while varying the laser frequency and external fields simultaneously in such a way as to keep $\epsilon$ and $f$ constant. According to closed orbit theory, such a spectrum is a smooth background plus a sum of sinusoidal oscillations,

$$
\sigma(w)=C_{0}+\sum_{k} C_{k} \sin \left(\tilde{S}_{k} w-\gamma_{k}\right),
$$

where $C_{0}$ is the absorption in the absence of recurrences, the sum is over classical closed orbits, $C_{k}$ is the recurrence amplitude of orbit $k$, and $\gamma_{k}$ is an additional phase related to the Maslov index. All of the quantities in Eq. (2) are constant or slowly varying across a scaled spectrum, except for $w$. Thus, the absolute square of the Fourier transform of a scaled spectrum with respect to $w$ produces a recurrence spectrum, which has a peak of height $\left|C_{k}\right|^{2}$ (the "recurrence strength") at the scaled action $\tilde{S}_{k}$ of each classical closed orbit.

The GTE recurrence reported in this paper, the lowestaction peak in these recurrence spectra, results from the family of "rotator"-type orbits that lie in the $z=0$ plane. Figure 1(a) shows this recurrence peak for a range of scaled electric fields, exhibiting the essential signatures of symmetry breaking and the transition from 2 to 3 nonseparable degrees of freedom. The falloff and revival behavior, qualitatively observed also for other rotator and vibrator type orbits, can be explained by a semiclassical theory of symmetry breaking.

Classical and semiclassical symmetry breaking. - As explained in the introduction, the perpendicular electric field destroys the cylindrical symmetry of hydrogen in a magnetic field. It can be shown that from each family $k$ of formerly closed orbits, just two isolated orbits return to the origin when $f \neq 0$ [see Figs. 2(a) and 2(b)]. From a classical perspective, therefore, one might expect a sudden drop in the recurrence strength when the electric field is

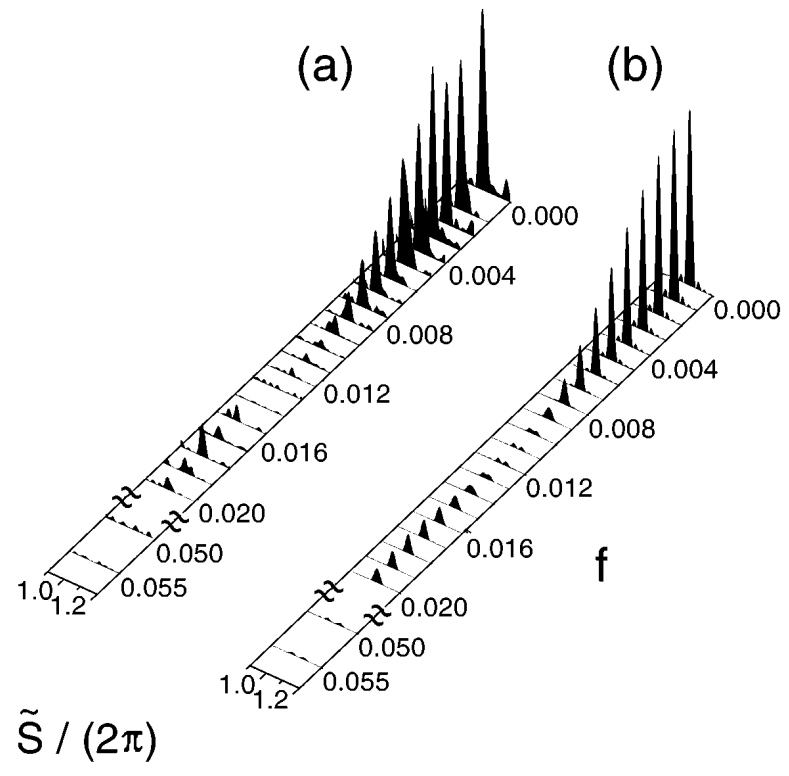

FIG. 1. These segments of (a) experimental and (b) theoretical scaled action spectra show how cylindrical symmetry breaking affects the strength of the Garton-Tomkins-Edmonds recurrence. The data are for deuterium at constant scaled energy $\epsilon=-0.15$, where the classical dynamics is fully chaotic. $B$ was scanned from 1 to $6 \mathrm{~T}$, i.e., $61.7 \geq w \geq 34.0$. The GTE recurrence peak is shown for several values of the (scaled) strength of the perpendicular electric field (the vertical scale is arbitrary). The recurrence is strongest when $f=0$, where it is enhanced by perfect focusing due to cylindrical symmetry. As the electric field is increased, the strength falls off quickly, vanishes, then increases again in a weak revival about $10 \%$ of the original peak height. (Note that there is a break in the $f$ axis.)

turned on. However, electron waves, unlike classical trajectories, diffract. Therefore, the whole family of returning trajectories, even those that miss the atom, still contributes to the recurrence. But waves arriving from different directions have different phases, so their interference is partially destructive, and the strength of the recurrence is reduced.

The phase of the returning wave from each direction in the $k$ th family is given by the classical action of the orbit returning from that direction. After symmetry is broken, that action $S_{k}^{F}(\phi)$ (here measured to the orbit's perihelion) depends on $\phi$, the orbit's azimuthal launching angle, and on $F$, the electric field strength. A theorem of classical mechanics asserts that the change in action caused by the electric field is proportional to the time integral of the perturbing Hamiltonian over the unperturbed trajectory: $\partial S_{k}^{F} / \partial F=-\int(\partial H / \partial F) d t$. It follows that, to first order in $F$, the actions of orbits within a family have a sinusoidal dependence on $\phi$ [13]:

$$
\begin{aligned}
S_{k}^{F}(\phi) & =S_{k}^{F=0}+\mathbf{F} \cdot \mathbf{d}_{k}(\phi) T_{k}, \\
& =S_{k}^{F=0}+F d_{k}^{\perp} T_{k} \cos \left(\phi-\phi_{k}^{1}\right),
\end{aligned}
$$

(see Fig. 3) where $\mathbf{d}_{k}(\phi) \equiv\langle-\mathbf{r}(t)\rangle$ is the time-averaged dipole moment of the orbit starting in direction $\phi, d_{k}^{\perp}$ is 

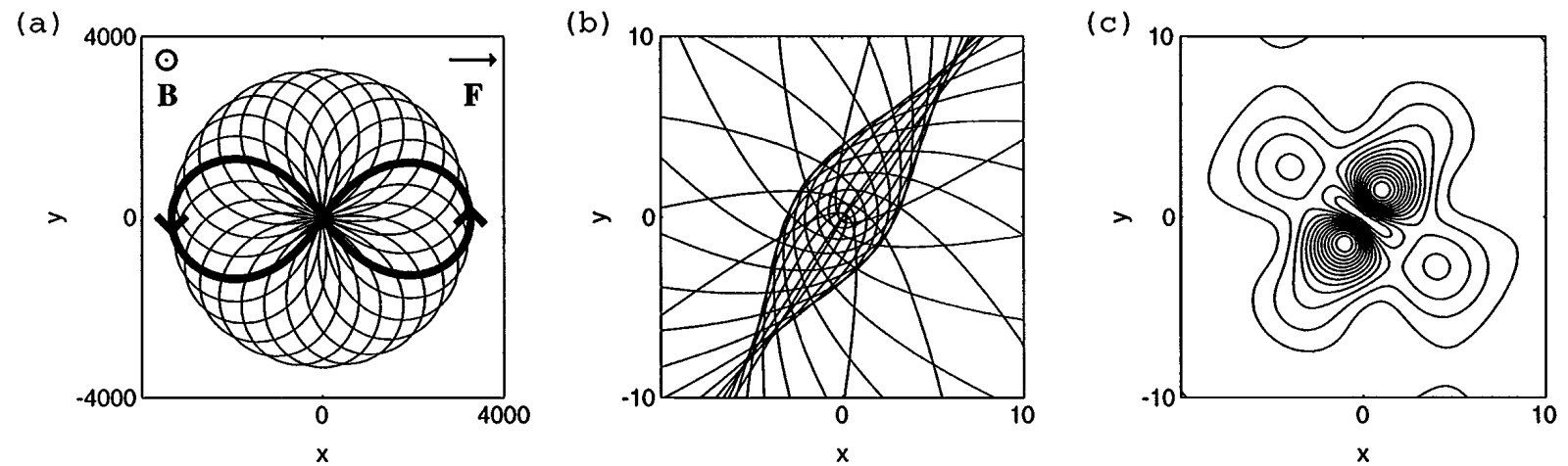

FIG. 2. Even a slight perpendicular electric field destroys the classical cylindrical symmetry. These figures were computed for the parameters $\epsilon=-0.15, f=0.012$, and $B=3 \mathrm{~T}$; distances are measured in Bohr radii. (a) The family of electron orbits associated with the GTE recurrence. These orbits lie in the $(x, y)$ plane, perpendicular to B. For nonzero electric field, only two orbits (shown with heavy lines) return exactly to the nucleus. (b) A closeup of the returning part of the trajectories as they approach the nucleus. The two closed orbits return to the nucleus diagonally; all others follow near-parabolic paths near the nucleus. (c) The symmetry-broken returning wave function $|\psi|^{2}$ associated with the same family of orbits, computed using the actions of Eq. (4). The wave function is generally large where the trajectories are dense, but destructive interference at the nucleus leads to a weak recurrence amplitude at this choice of parameters.

the magnitude of the component of $\mathbf{d}_{k}$ in the $x-y$ plane, and $T_{k}$ is the return time, all for the unperturbed orbit. The two closed orbits that survive the symmetry breaking are those with the extremal actions; their actions define the amplitude of the sinusoid.

The returning electron wave near the nucleus is a coherent superposition of waves arriving from each trajectory's final direction, each with a phase given by $\exp \left[i S_{k}^{F}(\phi)\right]$. The returning wave function for the GTE recurrence, calculated in this way, is shown in Fig. 2(c).

Falloff of recurrence strength. - A detailed analysis shows that the recurrence amplitude for weakly broken cylindrical symmetry is the coherent sum of contributions from each orbit in the family. Each orbit contributes nearly the same amplitude, but with a phase that varies according to Eq. (4). The net result is that Eq. (2) is replaced with



FIG. 3. Action $S(\phi)$ vs starting angle $\phi$ for the GTE family of orbits, for four different scaled electric field strengths. Action is measured at the orbit's closest approach to the nucleus, in units of $\hbar$. Actual values (symbols) are compared with the classical perturbation theory estimate of Eq. (4) (curves). For $f=0$ (cylindrical symmetry), the action is independent of $\phi$. The two surviving orbits occur at the indicated angles $\phi^{i}$ and $\phi^{2} \approx \phi^{1}+\pi$.

$$
\begin{aligned}
\sigma^{F}= & C_{0}+\sum_{k} \sigma_{k}^{F}, \text { where } \\
\sigma_{k}^{F} & =C_{k}^{F=0}\left\{\frac{1}{2 \pi} \int_{0}^{2 \pi} d \phi \sin \left[S_{k}^{F}(\phi)-\gamma_{k}\right]\right\}, \\
& =C_{k}^{f=0} J_{0}\left(f \tilde{d}_{k}^{\perp} \tilde{T}_{k} w\right) \sin \left(\tilde{S}_{k}^{f=0} w-\gamma_{k}\right) .
\end{aligned}
$$

The integral in Eq. (5) is over the orbits of family $k$, parametrized by their initial azimuthal angles $\phi$; i.e., this is the coherent superposition of waves having phases that vary with $\phi$. Equation (6) follows from substituting Eq. (4) into Eq. (5), evaluating the integral, and converting to scaled variables.

We see that each oscillation in the absorption spectrum is multiplied by a Bessel function that accounts for the partially destructive interference of the contributing orbits. The Fourier transform of Eq. (6) over the experimental range of $w$ predicts the height and shape of each peak in the recurrence spectrum. This theoretical prediction, evaluated for the GTE recurrence, is shown in Fig. 1(b). It is seen that theory explains all of the characteristics seen in experiment: the pattern of the falloff, the location of the minimum, and the location and relative strength of the revival.

It is to be noted that Eq. (5) is applicable through the whole transition - from perfect cylindrical symmetry to fully broken symmetry. In the former case $(f=0)$, the integrand in Eq. (5) is constant and the recurrence gives only the usual sinusoidal modulation of the absorption. In the latter case ( $f$ large), the integral can be evaluated by the method of stationary phase, and the result is the contribution from the two isolated returning orbits (including correct Maslov indices). The revival occurs when their phase difference reaches $2 \pi$ and they again interfere constructively.

In conclusion, the most important result of this work is an illumination of the difference between classical 
and quantum mechanical symmetry breaking. Classically, even the slightest symmetry breaking destroys the perfect focus, leaving only two surviving closed orbits, and reducing the density of returning trajectories instantaneously from infinite to finite. For strong symmetry breaking, only the two surviving closed orbits contribute significantly. However, in quantum mechanics there is an intermediate regime of weak symmetry breaking for which the contributions of the whole family of orbits - not just the ones that remain closed-must be considered together.

In this regime, the strength of the recurrence is determined by the degree of constructive and destructive interference across the whole returning wave. The strong recurrence for exact symmetry corresponds to pure constructive interference, and the minimum and revivalpurely nonclassical effects - are caused by the dominance of destructive and constructive interference, respectively, across the wavefront. This signature of symmetry breaking is seen in experiment and is predicted by a uniform semiclassical theory.

We thank Jörg Main for discussions of related quantum calculations. The work at the University of Bielefeld was supported by the Deutsche Forschungsgemeinschaft. The work at the College of William and Mary was supported by the Office of Naval Research and the National Science Foundation.

*Current address: Chemistry Department, Brookhaven National Laboratory, Upton, NY 11973.

${ }^{\dagger}$ Corresponding author.

[1] W. R. S. Garton and F. S. Tomkins, Astrophys. J. 158, 839 (1969).

[2] A. R. Edmonds, J. Phys. (Paris), Colloq. 31, C4 (1970).

[3] Magnetic: J. Main, G. Wiebusch, A. Holle, and K. H. Welge, Phys. Rev. Lett. 57, 2789 (1986); J. Main, G. Wiebusch, K. Welge, J. Shaw, and J. B. Delos, Phys. Rev. A 49, 847 (1994); T. van der Veldt, W. Vassen, and W. Hogervorst, Europhys. Lett. 21, 903 (1993).
[4] Electric: U. Eichmann, K. Richter, D. Wintgen, and W. Sandner, Phys. Rev. Lett. 61, 2438 (1988); M. Courtney, H. Jiao, N. Spellmeyer, D. Kleppner, J. Gao, and J. B. Delos, Phys. Rev. Lett. 74, 1538 (1995); J. Gao and J. B. Delos, Phys. Rev. A 49, 869 (1994).

[5] Parallel fields: J.-M. Mao, K. A. Rapelje, S. J. BlodgettFord, J.B. Delos, A. König, and H. Rinneberg, Phys. Rev. A 48, 2117 (1993); A. D. Peters, C. Jaffé, and J. B. Delos, Phys. Rev. Lett. 73, 2825 (1994); H. H. Fielding, J. Wals, W. J. van der Zande, and H. B. van Linden van den Heuvell, Phys. Rev. A 51, 611 (1995); M. Courtney, Phys. Rev. A 51, 4558 (1995).

[6] Crossed fields: G. Wiebusch, J. Main, K. Krüger, H. Rottke, A. Holle, and K.H. Welge, Phys. Rev. Lett. 62, 2821 (1989); G. Raithel, M. Fauth, and H. Walther, Phys. Rev. A 44, 1898 (1991); 47, 419 (1993); A. D. Peters and J. B. Delos, Phys. Rev. A 47, 3020 (1993); 47, 3036 (1993).

[7] M. L. Du and J. B. Delos, Phys. Rev. A 38, 1896 (1988); 38, 1913 (1988).

[8] E. G. Bogomol'nyí, Pis'ma Zh. Eksp. Teor. Fiz. 47, 445 (1988) [JETP Lett. 47, 526 (1988)]; Zh. Eksp. Teor. Fiz. 96, 487 (1989) [Sov. Phys. JETP 69, 275 (1989)].

[9] The three-dimensional version of closed orbit theory used by Raithel, Fauth, and Walther ([6], 1993 reference) applies if the electric field is sufficiently strong, but diverges as cylindrical symmetry is approached.

[10] A. M. Ozorio de Almeida, Hamiltonian Systems: Chaos and Quantization (Cambridge University Press, Cambridge, 1988); D. Ullmo, M. Grinberg, and S. Tomsovic, Phys. Rev. E 54, 136 (1996); S. C. Creagh, Ann. Phys. (N.Y.) 248, 60 (1996).

[11] Further details will be presented in a future publication.

[12] A. Holle, J. Main, G. Wiebusch, H. Rottke, and K.H. Welge, Phys. Rev. Lett. 61, 161 (1988).

[13] The one important exception is the orbit parallel to the magnetic field. That orbit has no dipole moment along $\mathbf{F}$, so the perturbation of the action is second order in the electric field. 